\title{
Importance of medium- to long-term therapeutic perspective in complementary and alternative medicine
}

\author{
Sayaka Mita ${ }^{1}$ and Takahiro Hayashi ${ }^{2 *}$ \\ ${ }^{1}$ Horticultural therapist, Division of Agricultural Science, Graduate school of Agriculture, Kindai University, Japan \\ ${ }^{2}$ Department of Agricultural Science, Faculty of Agriculture, Kindai University, Japan
}

\begin{abstract}
Integrative Clinical Medicine (ICM) is a combination of conventional medicine and complementary and alternative medicine (CAM). Since the therapeutic effect of CAM is slow and gradual, treatment requires a medium to long-term period. However, there is no mention of CAM's treatment strategy, that is, the perspective of planning and rationalizing treatment. We used a system dynamics model to express a group of factors related to a patient's therapeutic goals. Then, using that model, we constructed a method to proceed with treatment while predicting the therapeutic effect. With this method, it is not necessary to fumble for long-term treatment, and the effect over time can be confirmed dynamically and quantitatively as in the PDCA cycle. Here, we would like to comment on the importance of a medium- to long-term treatment strategy perspective in CAM.
\end{abstract}

Currently, medical treatment methods are diversifying. Conventional treatments have primarily aimed to cure a patient's illness through medication, radiation, and surgery. On the other hand, recent treatment methods are trying to provide comprehensive care for patients from the viewpoint of disease prevention, rehabilitation, and long-term care in order to lead patients to a healthy and happy life. Complementary therapy is the addition of another treatment to the conventional main medical treatment. Alternative therapies replace the main treatment with a different therapy. These treatment methods are collectively called complementary and alternative medicine (CAM), and the number of cases used clinical CAM treatment is increasing [1].

Integrative Clinical Medicine (ICM) is a combination of CAM and conventional medicine. The concept of ICM is to treat the whole body by combining multiple treatments. ICM treats the entire body from a holistic perspective, rather than treating the affected area partially. The authors have been studying horticultural therapy, one of the CAMs, for many years. Scientific evidence is needed to use this treatment as a CAM in clinical practice [2]. However, this treatment is not an immediate treatment like conventional medicine and requires a different medical strategy. This paper explains the importance of medium- to long-term therapeutic perspectives that medical doctors and therapists should have at CAM.

The therapeutic effect of CAM is slow, and it takes a long treatment period

CAM is a traditional health care that people have used to maintain their health in their daily lives. This treatment includes aromatherapy, naturopathic medicine, acupuncture, and oriental medicine (traditional Chinese medicine), all of which have slow-acting therapeutic effects. CAM is characterized by supporting the health of patients through continuous and long-term intervention rather than temporary intervention, and treatment takes time.
Clear and cost-effective medical benefits are also required in CAM

Although it depends on the medical system of each country, many CAM treatment methods are not covered by health insurance, and medical expenses are often high. Patients understand the advantages of CAM, which has no side effects and makes the mind and body healthy overall but demand a rational explanation of the medical effects of CAM in terms of cost effectiveness. The medical doctor or therapist needs to give due consideration in advance to the mechanism of action of CAM and the time required for treatment.

\section{CAM requires a medium- to long-term treatment strategy perspective}

The therapeutic effect of CAM is slow and appears slowly and gradually. Medical doctors and therapists will work on medium- to long-term treatment while objectively evaluating the effects without overlooking them. Here, it is assumed that the medium term is half a year and the long term is one year or more. During the course of treatment, doctors and therapists need to confirm the effectiveness of the treatment they have given and explain the process to the patient. Since conventional medicine has an immediate effect, the importance of formulating a long-term medical strategy using a small integrated therapeutic effect as an index has not been pointed out so far. The

${ }^{\star}$ Correspondence to: Takahiro Hayashi, Floriculture Laboratory, Department of Agricultural Science, Faculty of Agriculture, Kindai University, 3327-204 Nakamachi, Nara City 631-8505, Japan, E-mail: thayashi@nara.kindai.ac.jp

Key words: complementary and alternative medicine, horticultural therapy, system dynamics model, treatment strategy

Received: September 15, 2020; Accepted: September 27, 2020; Published: September 30, 2020 
therapeutic effect is objectively indexed as a variable with respect to time. Variables include quantitative variables and qualitative variables. Quantitative variables are numerical data obtained by analysis with medical devices, so they are easy to use as indicators of effectiveness. Qualitative variables are descriptive data in characters, and in this case, it is necessary to convert them into numerical values on an ordinal scale and make them objective. Although the indicators of therapeutic effect differ depending on the type of CAM, it is important in treatment to have a medium- to longterm perspective of improving the patient's condition through continuous intervention based on reproducible indicators.

\section{A perspective can be established by setting cartesian coordinates}

The long-term perspective of CAM can be explained using orthogonal coordinate axes. In many cases, the index of therapeutic effect is evaluated as a scale or numerical data, so it can be positioned as a variable. The course of treatment can be positioned as a time axis common to all CAMs. By setting the index of therapeutic effect on the vertical axis and the course of treatment on the horizontal axis, it is possible to easily understand where the patient's health condition is currently positioned. Furthermore, it is possible to infer how much therapeutic effect should be provided next in order to improve the health condition at that time. In order to enhance the long-term therapeutic effect, medical doctors and therapists set the coordinate axes of the therapeutic effect and the treatment course, and repeat planning, treatment, evaluation, and improvement for the patient's health condition like the PDCA cycle (Figure 1). Treatment can be reasonably advanced within the target period.

\section{Variables on the vertical axis serve as indicators of long-term treatment}

In recent years, it has been clarified that improvement of mental health promotes recovery of physical function. Self-efficacy, advocated by Bandura, is one of the indicators of mental health. Self-efficacy refers to the belief in recognizing motives and deciding actions for events and tasks that people perceive [3]. People with high self-efficacy try to act positively. In horticultural therapy, when the mind tries to act, the person moves the body. The illness is mindful, primarily important in horticultural therapy, and what needs to be improved is the human mind.

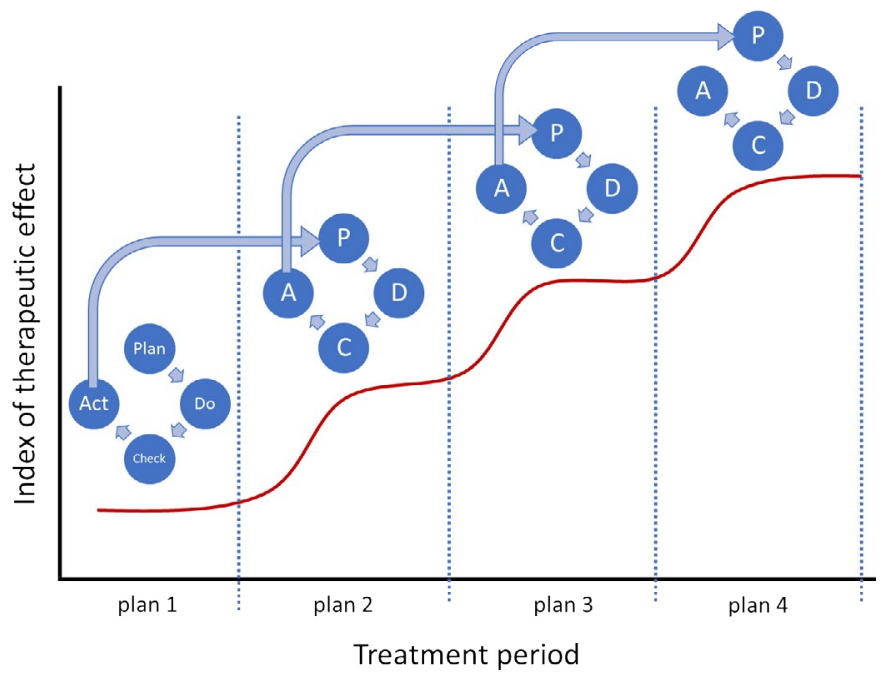

Figure 1. Medium- to long-term treatment strategy in CAM.

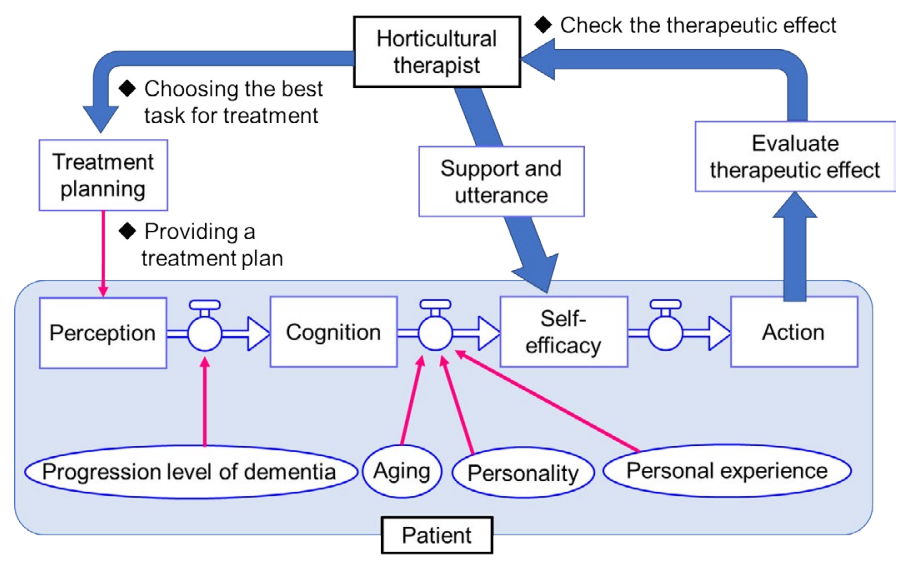

Figure 2. Horticultural therapy system dynamics model.

Self-efficacy represents a state of mind that seeks to live positively, and many researchers, including Bandura, have reported methods for scaling, or quantifying. Taking horticultural therapy as an example, the horizontal axis is the treatment period and the vertical axis is the quantified self-efficacy, so that the time course of the treatment effect can be graphically evaluated on Cartesian coordinates.

\section{Expressing the causal relationship of factors with system dynamics}

Patients' high self-efficacy is affected not only by therapeutic intervention but also by various factors such as age, personal and social experience, personality, and recovery of mental and physical functions. It suggests that therapeutic effects do not always have a positive effect on patient health but may have a negative effect under their influence. When performing treatment, the relationship between the therapeutic index and treatment intervention is emphasized, but the longer the treatment period, the more it is necessary to consider their effects. The causal relationship between multiple factors can be visually expressed by system dynamics.

System dynamics is a method that can quantitatively examine the factors that change over time and the relationships between the factors. In addition to therapeutic intervention, the long-term therapeutic effect of CAM involves various effects such as the patient's physical and mental condition, illness, aging, and social conditions.

Therefore, it is necessary to consider the relationship between these factors for the therapeutic effect as an overall system and create a treatment plan. We constructed a system dynamics model by replacing scales and numerical data of horticultural therapy effects, therapeutic interventions, and other factors as variables (Figure 2).

Numerical simulation is possible for system dynamics by using dedicated software such as Stella (paid) and Vensim (free). The authors performed an analysis using Stella and were able to predict the therapeutic effect of a long-term treatment plan corresponding to the effects of the patient's disease and aging to some extent. The therapeutic effect is shown in a graph that rises to the right in Cartesian coordinates, but it was possible to rotate the PDCA cycle while comparing the predicted value (target value) and the measured value, and it was possible to have a long-term perspective. By incorporating system dynamics into CAM and ICM, it is possible to plan treatment considering various factors that affect the health condition of patients, and it is thought that the therapeutic effect can be enhanced. 


\section{Usefulness of system dynamics as a perspective of medium- to long-term medical strategy}

The application of system dynamics to CAM and ICM can facilitate information sharing with experts in other fields. System dynamics can quantitatively indicate the patient's health condition and therapeutic effect. It facilitates understanding of therapeutic effects beyond the differences in knowledge and skills that arise from different disciplines. The system dynamics predictions may also be useful when considering how often conventional medicine and CAM are provided to patients as the most appropriate treatment. In the future, in order to use CAM and ICM to which system dynamics are applied in clinical practice, it is necessary to verify the variables that compose them and the numerical values of interactions between variables to improve the accuracy of prediction results.

\section{References}

1. Ventola CL (2010) Current Issues Regarding Complementary and Alternative Medicine (CAM) in the United States Part 1: The Widespread Use of CAM and the Need for Better-Informed Health Care Professionals to Provide Patient Counseling. Pharmacol Ther 35: 461-468. [Crossref]

2. Kantor M (2009) The Role of Rigorous Scientific Evaluation in the Use and Practice of Complementary and Alternative Medicine. J Am Coll radiol 6: 254-262. [Crossref]

3. Bandura A (1997) Self-efficacy: The exercise of control. New York: W. H. Freeman.

Copyright: $@$ 2020 Mita S. This is an open-access article distributed under the terms of the Creative Commons Attribution License, which permits unrestricted use, distribution, and reproduction in any medium, provided the original author and source are credited. 